$01,07,19$

\title{
Связь макроскопических характеристик твердого тела с энергией связи иона в решетке металлов
}

\author{
(C) К.М. Ерохин ${ }^{1}$, Н.П. Калашников ${ }^{2}$ \\ ${ }^{1}$ Московский политехнический университет, \\ Москва, Россия \\ ${ }^{2}$ Национальный исследовательский ядерный университет „МИФИ“, \\ Москва, Россия \\ E-mail: kalash@mephi.ru
}

Поступила в Редакцию 15 февраля 2021 г.

В окончательной редакции 2 февраля 2021 г.

Принята к публикации 2 февраля 2021 г.

Рассмотрена связь между макроскопическими параметрами, такими как модуль Юнга в законе Гука, скорость звука и температура Дебая, и энергией связи отдельного атома. Предложена формула для расчета модуля упругой деформации. Получено соотношение между скоростью звука и энергией связи отдельного атома в твердом теле. Установлена простая формула для расчета скорости звука в металлическом стержне. Предлагается связь характеристической температуры Дебая с энергией связи иона в решетке твердого тела.

Ключевые слова: упругая деформация, модуль Юнга, закон Гука, скорость звука, характеристическая температура Дебая, энергия связи атома, концентрация атомов.

DOI: 10.21883/FTT.2021.07.51030.031

\section{1. Введение}

В. Вайскопф в серии работ „Просто о сложном“ [1] описал возможность микроскопической интерпретации некоторых макроскопических характеристик веществ. Мы предлагаем рассмотреть процесс упругой деформации вещества и связать макроскопическую характеристику, модуль Юнга, с энергией связи отдельного атома и концентрацией атомов в единице объема вещества. В настоящей работе продолжена серия оценок, позволяющих связать макроскопические характеристики такие как, модуль упругой деформации, скорость звука и характеристическая температура Дебая, с энергией связи отдельного атома и концентрацией атомов в единице объема вещества.

\section{2. Процесс упругой деформации вещества}

Закон Гука предполагает, что упругая сила деформации пропорциональна относительному изменению длины стержня [2]:

$$
\frac{F}{S}=\sigma=E \frac{\Delta x}{x},
$$

где $\sigma$ - напряжение в стержне, $E-$ модуль Юнга, $\Delta x / x-$ относительное удлинение стержня.

Упругая деформация стержня приводит к увеличению потенциальной энергии $U$ единицы объема стержня $[2,3]$ :

$$
\frac{\Delta U}{\Delta V}=\frac{\sigma^{2}}{2 E}=\frac{E}{2}\left(\frac{\Delta x}{x}\right)^{2} .
$$

Допустим, что мы сообщаем стержню такую потенциальную энергию, что на каждый атом вещества прихо- дится энергия, равная энергии связи атома $\varepsilon_{b}-$ энергия, необходимая для отделения атома от вещества [3]).

Если бы мы применили такую операцию, то весь материал разделился бы на отдельные атомы, т.е. разрушился. Примем качественно, что такое разрушение происходит, когда межатомные расстояния увеличиваются в два раза, т. е. длина стержня удваивается: $\Delta x / x \approx 1$ !

\section{3. Связь модуля Юнга с энергией связи атома в решетке}

Рассмотрим поведение двух соседних атомов стержня, материал которого характеризуется концентрацией

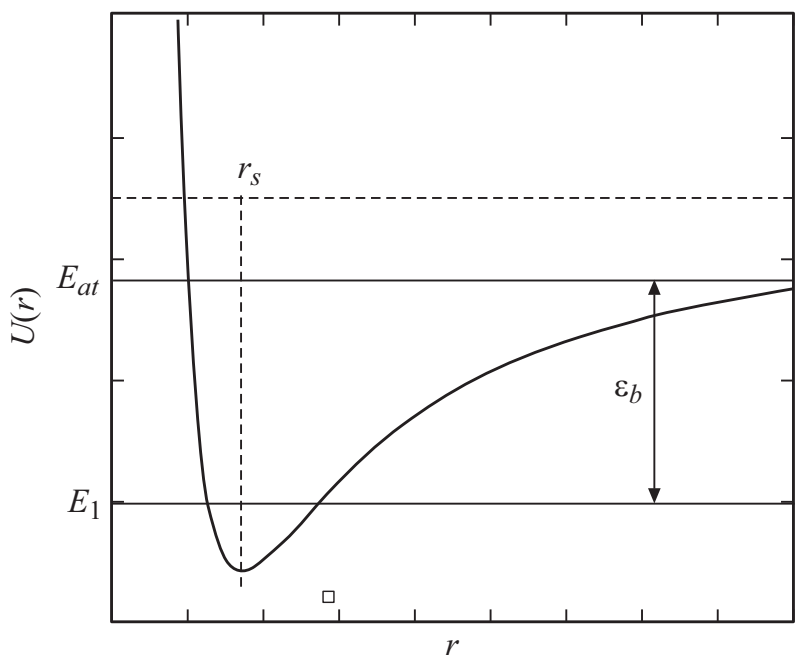

Рис. 1. Качественная зависимость полной энергии электрона проводимости от расстояния до центра ячейки. 
$n_{0}\left(10^{28} \mathrm{~m}^{-3}\right)$. При относительной деформации $\Delta x / x$ потенциальная энергия одного атома $(\Delta U)_{1}$ увеличивается на величину

$$
(\Delta U)_{1}=\frac{E}{2}\left(\frac{\Delta x}{x}\right)^{2} \frac{1}{n_{0}} .
$$

С другой стороны, увеличение потенциальной энергии для „максимально“ возможной деформации $\Delta x / x=1$ равняется $\varepsilon_{b}$. Таким образом, мы получаем оценку для модуля Юнга

$$
E=2 n_{0} \varepsilon_{b}
$$

На рис. 2 приведены результаты расчетов модуля Юнга для ряда металлов на основе формулы (4). Для сравнения приведены его экспериментальные значения в длинном стержне $[4,5]$ :

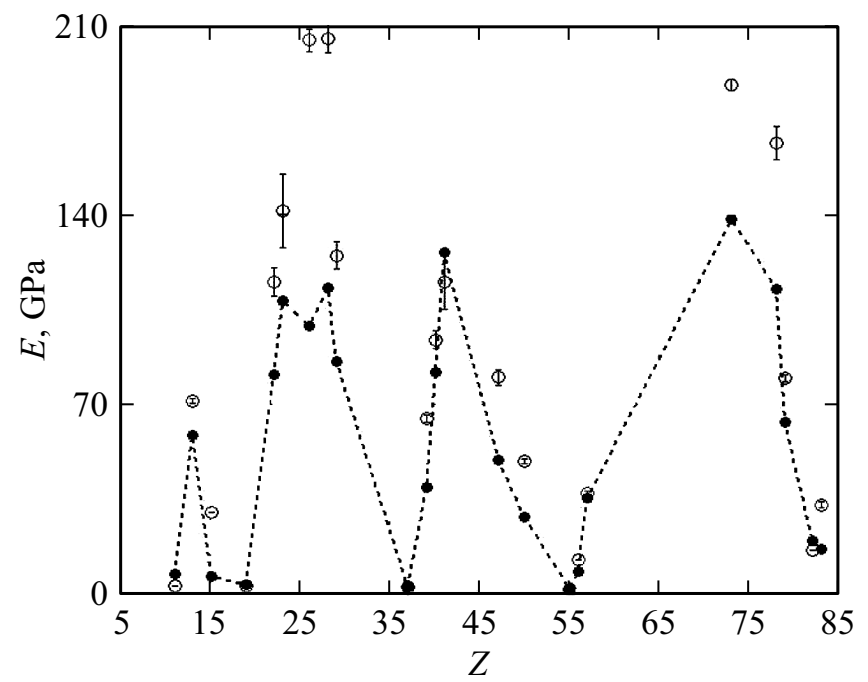

Рис. 2. Экспериментальные (светлые кружки) $[4,5]$ и расчетные (темные кружки) значения модуля Юнга (4).

Как видно из рис. 2, большинство простых твердых тел обладают модулем Юнга порядка сотни GPa.

Результаты расчетов дают оценку коэффициента корреляции между расчетными и экспериментальными данными 0.95. Таким образом, макроскопический параметр как степень жесткости материала определяется микроскопическим параметром, таким как удельная энергия связи иона в кристаллической решетки.

Для подтверждения этого предположения, на рис. 3 приведена связь между теоретическими (4) и экспериментальными значениями модуля Юнга.

Как следует из рис. 3, связь между ними хорошо описывается линейной регрессией. Полученные различия определяются тем фактом, что не было принято во внимание различия симметрий кристаллических решеток элементов.

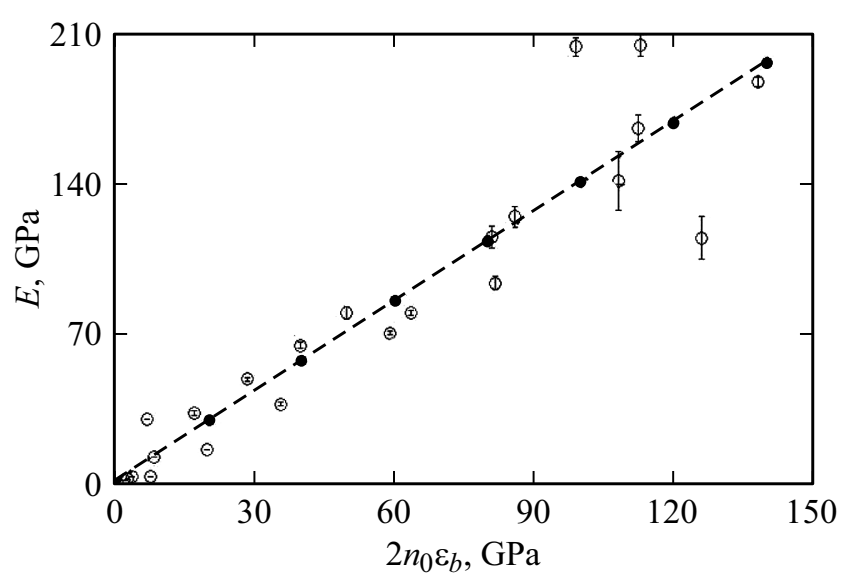

Рис. 3. Экспериментальные значения (светлые кружки) модуля Юнга $[5,6]$ в зависимости от энергии связи иона в решетке.

\section{4. Связь скорости звука с энергией связи отдельного атома в твердом теле}

Одним из важных макроскопических свойств металлов является скорость распространения в нем звука. Упругие колебания, возбужденные в какой-либо точке среды, распространяются в ней с конечной скоростью, зависящей от индивидуальных особенностей этой среды. Скорость звука в изотропных твердых телах определяется модулями упругости вещества. Так для стержня - тела, длина которого значительно превышает его поперечные размеры, но (по порядку) не меньше длины волны упругих колебаний, скорость звука $[2,7]$ составляет

$$
V_{s}=\sqrt{\frac{E}{\rho}},
$$

где $E-$ модуль Юнга, $\rho$ - плотность вещества.

Подстановка (4) в выражение (5) определяет соотношение между скоростью звука в стержне, состоящем из элемента с атомной массой иона решетки $A$ и энергией связи $\varepsilon_{b}$

$$
V_{s}=\sqrt{2 \frac{N_{A}}{A} \varepsilon_{b}}
$$

где $N_{A}$ - число Авогадро, $A$ - атомная масса.

На рис. 4 представлены результаты расчетов скорости звука по формуле (6) и справочными данными $[4,8,9]$ для ряда элементов периодической системы в зависимости от атомного номера $Z$. Как видно из рис. 4 имеет место достаточно хорошее соответствие между расчетными и соответствующими справочными данными. Следует еще раз отметить, что в расчетах не учитывались индивидуальные симметрии кристаллических решеток металлов.

С целью дополнительной проверки формулы (6) были проведены вычисления скорости звука $V_{s}$ на основе формулы (6) и сравнение с табличными значениями работ $[4,5,9]$. На рис. 5 представлена зависимость расчетных (6) и экспериментальных данных $[4,5,9]$ для скорости звука в зависимости от энергии связи и атомной 


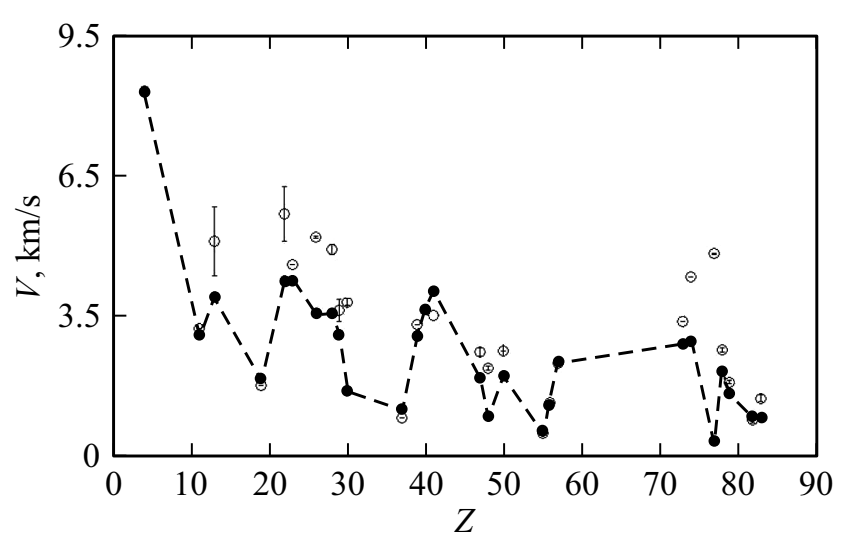

Рис. 4. Табличные (светлые кружки) $[4,8,9]$ и расчетные (темные кружки) значения (6) скорости звука.

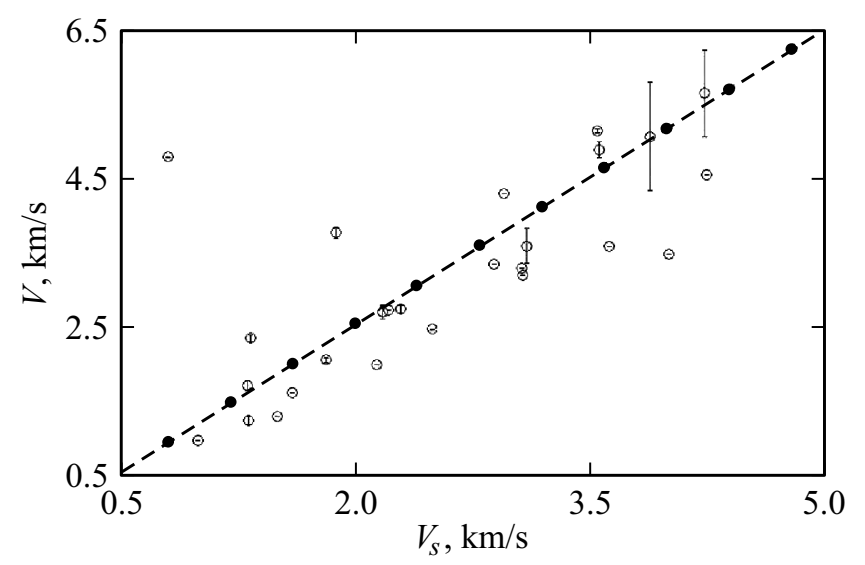

Рис. 5. Зависимость скорости звука в стержне от энергии связи иона металла (6).

массы ионов в металлическом стержне. Как видно из этого рисунка связь между этими величинами хорошо представляется линейной регрессией с коэффициентом корреляции 0.89 .

Следует отметить, что в работе [10] предпринималась попытка связать скорость звука в твердом теле с фундаментальными физическими константами.

\section{5. Связь характеристической температура Дебая с энергией связи отдельного атома в твердом теле}

Значения характеристической температуры Дебая определяется значениями максимальных частот акустических колебаний кристаллической решетки

$$
T_{D}=\frac{\hbar \omega_{D}}{k_{B}}
$$

где $\omega_{D}-$ максимальная частота упругих колебаний кристаллической решетки; $\hbar-$ постоянная Планка; $k_{B}-$ постоянная Больцмана. Температура Дебая $T_{D}$ разграни- чивает области низких и высоких температур по отношению к решеточным свойствам кристалла [11]. При температурах, меньших температуры Дебая $\left(T<T_{D}\right)$, некоторые колебания уже не возбуждаются, т.е. некоторые моды начинают „вымерзать“, проявляются квантовые эффекты и необходимо использование квантовой статистики. При температуре, большей температуры Дебая $\left(T>T_{D}\right)$, возбуждаются все возможные колебания, при этом справедлива классическая статистика. Величина $k_{B} T_{D}$ представляет собой максимальный квант энергии, способный возбудить колебания решетки.

Температура Дебая характеризует многие свойства металлов - от микроскопических характеристик энергии активации металлов - до макроскопических характеристик - теплоемкости, электропроводности и др. Согласно [12] максимальная частота нормальных колебаний

$$
\omega_{D}=\left(6 \pi^{2}\right)^{1 / 3} \frac{V_{s}}{r_{s}},
$$

параметр $r_{s}$ - постоянная решетки, величина которой определяется положением минимума потенциальной энергии (см. рис. 1).

Следуя работе [12], выполним подстановку (6) и (8) в формулу (7), что позволяет получить связь между температурой Дебая и микроскопическими характеристиками твердого тела

$$
T_{D}=\left(6 \pi^{2}\right)^{1 / 3} \frac{\hbar}{k_{B} r_{s}} \sqrt{2 \frac{N_{A}}{A} \varepsilon_{b}} .
$$

Каждую кристаллическую структуру можно охарактеризовать определенным набором элементарных трансляций. В зависимости от отношения значений и взаимной ориентации основных трансляций $a, b, c$ получаются решетки, отличающиеся друг от друга по своей симметрии. Вигнером и Зейтцем был предложен способ построения таких примитивных ячеек, которые обладали бы полной симметрией решетки Бравэ. Если выбрать в качестве элементарной ячейки прямого пространства ячейку Вигнера-Зейтца, то для плотноупакованных металлов граница элементарной ячейки является поверхностью с высокой степенью симметрии и потому может быть представлена сферой радиуса $r_{W Z}$ того же объема [11]. Каждая такая сфера содержит один узел решетки и является примитивной элементарной ячейкой. Поэтому, для оценки параметра $r_{s}$ будем полагать, что положение минимума кривой потенциальной энергии определяется радиусом такой сферы, т. е.

$$
r_{s} \approx r_{W Z}=\left(\frac{3}{4 \pi n_{0}}\right)^{1 / 3}
$$

С учетом приближения (10) был проведен расчет температуры Дебая по формуле (9). Результаты расчетов и сравнение их с табличными данными работ $[4,6,9]$ представлены на рис. 6.

Как видно из рис. 6 совпадение между расчетными и экспериментальными значениями достаточно хорошее. 


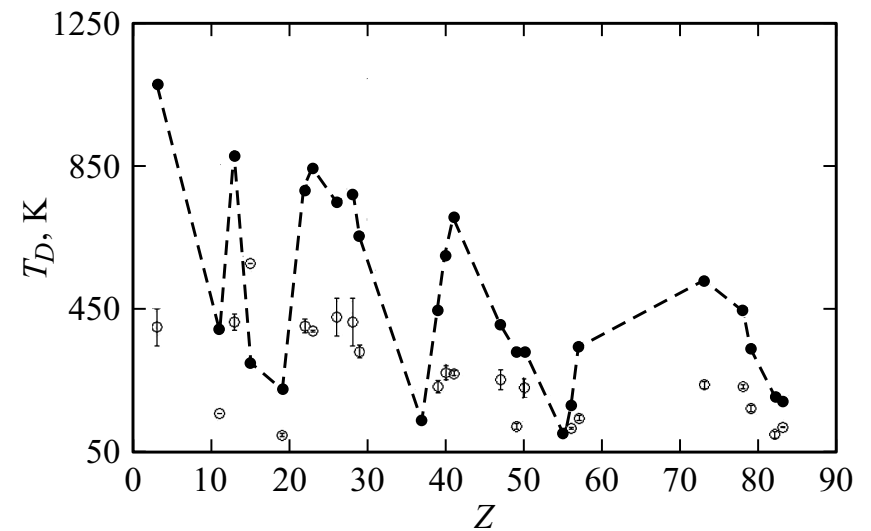

Рис. 6. Экспериментальные (светлые кружки) [4,9] и расчетные (темные кружки) значения (9) температуры Дебая в модели Вигнера-Зейца с параметром $r_{s}(10)$.

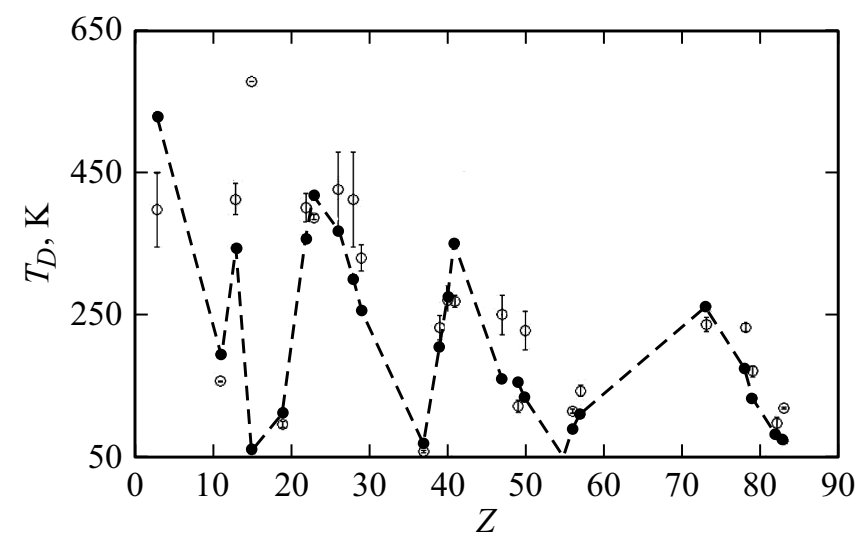

Рис. 7. Экспериментальные (светлые кружки) и расчетные (темные кружки) значения (9) температуры Дебая в модели аппроксимации всех индивидуальных симметрий кубической сингонией.

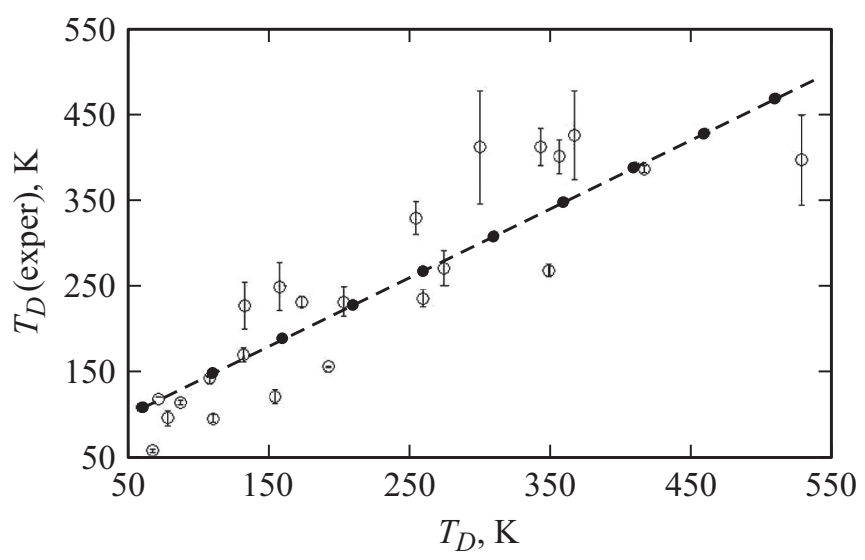

Рис. 8. Сравнения вычисленных по формуле (9) значений температур Дебая $T_{D}$ с табличными значениями $[4,9] T_{D}$ (exper).

Так, среднее расстояние между ними составляет около 59 К. Результаты можно изменить в лучшую сторону, если сделать предположение, что любую сингонию кри- сталла можно заменить на кубическую с сохранением их объемов, равных $r_{s}^{3}$. Экспериментальные данные по параметрам решетки аппроксимировались кубической сингонией с сохранением объема решетки. Результаты расчетов с использованием такого приближения представлены на рис. 7.

Результаты расчетов по формуле (9) значений температур Дебая и сравнение их табличными данными представлены на рис. 8. Штриховая линия регресии указывает на существование прямой зависимости между этими величинами.

\section{6. Заключение}

В настоящей работе на основе единого микроскопического подхода, предложенного в работах Вайскопфа [1], был сделан анализ макроскопических параметров, таких как модуль Юнга, характеризующий свойство материалов сопротивляться растяжению и сжатию при упругой деформации, скорости звуковой волны, которая определяется макроскопическими характеристиками среды распространения, и температуры Дебая, определяющей область применения классических приближений. Показано, что все эти параметры не являются независимыми, а определяются величиной энергии связи иона в кристаллической решетке.

Несмотря на приближенный характер используемой модели, в рамках подхода Вайскопфа, были получены простые соотношения между этими параметрами и основной характеристикой металлов - энергией связи. Такие соотношения подтверждаются хорошим соответствием между расчетом и табличными или экспериментальными данными. Полученные зависимости позволяют с единой точки зрения рассматривать различные макроскопические характеристики простых металлов.

\section{Благодарности}

К.Н.П. благодарен Программе конкурентоспособности НИЯУ МИФИ за поддержку исследований.

\section{Конфликт интересов}

Авторы заявляют, что у них нет конфликта интересов.

\section{Список литературы}

[1] V.F. Weisskopf, H. Bernstein. Amer. J. Phys. 53, 12, 1140 (1985).

[2] Л.Д. Ландау, Е.М. Лифшиц. Теория упругости. Наука, М. (1965). T. 7. 203 c.

[3] Ч. Киттель. Введение в физику твердого тела. Наука, М. (1978). $791 \mathrm{c}$.

[4] Физические величины. Справочник / Под ред. И.С. Григорьева, У.3. Мейлихова. Энергоатомиздат, М. (1991). $1232 \mathrm{c}$. 
[5] Smithsonian Physical Tables. $9^{\text {th }}$ Rev. Ed. / W.E. Forsythe. Norwich, N.Y. (2003). P. 785.

[6] P.A. Freshney. Education. Periodic Table. V. 3.9. https:/www.softpedia.com/get/Others/Home-Education/ Freshney-Periodic-Table.shtml

[7] Дж. Займан. Принципы теории твердого тела. Мир, M. (1974). 472 c.

[8] C.Y. Ho, R.W. Powell, P.E. Liley. J. Phys. Chem. Ref. Data, 3. Suppl. 1, I796 (1974).

[9] https://periodictable.com/Properties/A/SoundSpeed.html.

[10] K. Trachenko, B. Monserrat, C.J. Pickard, V.V. Brazhkin. Sci. Adv. 6, 41, 5 (2020).

[11] Н.А. Ашкрофт, Н. Мерлин. Физика твердого тела. Мир, M. (1979). T. 2. 422 c.

[12] Н.П. Калашников, М.А. Смондырев Основы физики. Бином. М. Лаборатория знаний (2017). Т. 2. 606 с.

Редактор Ю.Э. Китаев 